INFORMAL REPORT

Neutrino Disintegration of the Deuteron at LAMPF Energies 
This report was prepared as an account of work sponsored by the United States Government. Neither the United States nor the United States Atornic Energy Commission, nor any of their empleyees, nor any of their contractors, subcontractors, or their employees, makes any warranty, express or implied, or assumes any legal liability or responsibility for the accuracy, com pleteness or usefulness of any information, apparatus, product or process disclosed, or represents that its use would not infringe privately owned rights.

In the interest of prompt distribution, this LAMS report was not edited by the Technical Information staff.

Printed in the United States of America. Available from National Technical Information Service

U. S. Department of Commerce

5285 Port Royal Road

Springfield, Virginia 22151

Price: Printed Copy $\$ 3.00$; Microfiche $\$ 0.95$ 
LA-5175-MS

Informal Report

UC-34

ISSUED: March 1973

\title{
Neutrino Disintegration of the Deuteron at LAMPF Energies
}

by

\author{
J. S. O'Connell*
}

*Consultant. Permanent Address: National Bureau of Standards, Washington, D. C.

NOTICE

This report was prepared as an account of work
sponsored by the United States Government. Neither
the United States nor the United States Atomic Energy
Commission, nor any of their employees, nor any of
their contractors, subcontractors, or their employees,
makes any warranty, express or implied, or assumes any
legal liability or responsibility for the accuracy, com-
pleteness or usefulness of any information, apparatus,
product or process disclosed, or represents that its use
would not infringe privately owned rights.

would not infringe privately owned rights. 
NEUTRINO DISTNTEGRATIYY OF TEE DEJTERON

AT LAMPF ENERGIES

3

J. s. c'rconell

\section{ABSTRACT}

The differential cross section for the neutrino disintegration of the deuteron is calculated using effective range theory for neutrino ezergies From zero to $53 \mathrm{MeV}$.

The general formula for the neutrino-nucleus reaction

$$
v_{e}+A(Z, N) \rightarrow A(Z+1, N-1)+e^{-}
$$

with the kinematir.s defined as

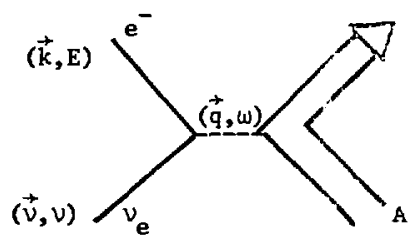

is given by

$$
\dot{=} \sigma=2 \tau \frac{d n}{d v} \sum_{\substack{\text { lefton } \\ \text { spins }}} \frac{1}{2 J_{1}+1} \sum_{m_{f}} \sum_{m_{i}}\left|\left\langle f\left|\hat{H}_{w}\right| 1\right\rangle\right|^{2} .
$$

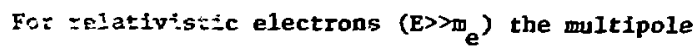
cecomisition of the weak interaction Hamiltonian $b=$ weer nuciear states of definite total angular ti dertur ard parity is $(1,2)$

$$
\begin{aligned}
& \sum_{\substack{\text { lepton } \\
\text { spins }}} \frac{1}{2 J_{i}+I} \sum_{m_{f}} \sum_{m_{i}}\left|\left\langle f\left|\hat{H}_{w}\right| i\right\rangle\right|^{2}=2 \mathrm{c}^{2} \frac{4 \pi}{2 J_{i}+I} \cos ^{2} \vdots \sum_{i=j}\left|\left\langle J_{f}|| M_{J}+\frac{\omega}{q} L_{J}|| J_{i}\right\rangle\right|^{2}
\end{aligned}
$$

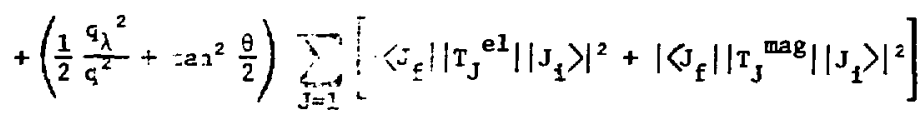

$$
\begin{aligned}
& \left.-\tan \frac{\theta}{2}\left(\frac{q_{i}^{2}}{q^{2}}+\tan ^{2} \frac{\theta}{2}\right)^{\frac{1}{2}} \sum_{J=I} 2 \operatorname{Re}\left\langle J_{E}\left\|T_{J}{ }^{m a g}\right\| J_{I}\right\rangle\left\langle J_{E}\left\|T_{J} e 1_{1}\right\| J_{f}\right\rangle^{*}\right\}
\end{aligned}
$$

$$
\text { where } \cos \theta=\hat{k} \cdot \hat{v} \text { and } q_{\lambda}^{2}=q^{2}-\omega^{2} \text {. }
$$


These multipole operators are the partfal-wave decompositions of the operators

$$
\tau_{ \pm}(1, \vec{\sigma}, \vec{p}, \vec{\sigma} \cdot \vec{p}) e^{i \vec{q} \cdot \vec{r}}
$$

They are definct in references (1) and (2) and are made up of spherisal bessel functions in qr, vector sphertcal harmonics, and the nucleon spin and momentum operators.

The cross section for the aucleon reaction

$$
\nu_{e}+N \rightarrow P+e^{-}, Q=.78 \mathrm{MeV}
$$

is evaluated by taking the denrity of final states as

$$
\frac{d n}{d \nu}=\frac{d^{3} k}{(2 \pi)^{3} d \nu}=\frac{k E d \Omega e}{(2 \pi)^{3}}
$$

and evaluating the reduced matrlx elements $\left(J_{1}=\frac{1}{2}\right.$, $J_{f}=\frac{1}{2}$ ) of the major low momentum transfer terms, viz., the vector,

$$
\begin{aligned}
\left\langle\frac{1}{2}|| M_{0}^{+} \frac{\omega}{q} L_{0}|| \frac{1}{2}\right\rangle & =\left\langle\frac{1}{2}|| f_{0}(q r) Y_{0}\left(\Omega_{r}\right)|| \frac{1}{2}\right\rangle \\
& =\left(\frac{2}{4 \pi}\right)^{\frac{1}{2}}\left(1-\frac{\omega^{2}}{q^{2}}\right) f_{N}(q)
\end{aligned}
$$

and the axial vector (with subscript 5 from $\gamma_{s}$ )

$$
\begin{aligned}
\left\langle\frac{1}{2}|| T_{1}^{e l^{5}}|| \frac{1}{2}\right\rangle & =F_{A}\left(\frac{2}{3}\right)^{\frac{1}{2}}\left\langle\frac{1}{2} \|_{0}(q r) \vec{y}_{101} \cdot \cdot \vec{\sigma}|| \frac{1}{2}\right\rangle \\
& =F_{A} \frac{2}{\sqrt{4 \pi}} F_{N}(q)
\end{aligned}
$$

and the vector

$$
\begin{aligned}
\left\langle\frac{1}{2}|| \mathrm{T}_{1}^{\mathrm{mag}}|| \frac{1}{2}\right\rangle & =\left(\frac{2}{3}\right)^{\frac{1}{2}} \frac{q \mu_{\mathrm{y}}}{2 \mathrm{M}}\left\langle\frac{1}{2} \|_{0}(\mathrm{qr}){\overrightarrow{V_{101}}}_{10} \cdot \vec{\sigma}|| \frac{1}{2}\right\rangle \\
& =\left(\frac{q \mu_{\mathrm{v}}}{2 \mathrm{M}}\right) \frac{2}{\sqrt{4 \pi}} \mathrm{f}_{\mathrm{N}}(q)
\end{aligned}
$$

where $f_{N}(q)$ is the nucleon form factor and $\mu_{v}=$ $\mu_{p}-\mu_{n} 1 s$ the nucleon vector magnetic moment.
Substituting in Eqs. (1) and (2) gives

$$
\begin{array}{r}
\frac{d \sigma_{U N}}{d{ }_{e}}=\frac{G^{2} k E}{4 ?^{2}}\left\{2 \left(1-\frac{\left.\omega^{2}\right)^{2}}{q^{2}} \cos ^{2} \frac{\theta}{2}+4\left[F_{A}{ }^{2}+\left(\frac{q \mu v}{2 M}\right)^{2}\right]\right.\right. \\
\left.\left(\frac{1}{2}+\frac{1}{2} \sin ^{2} \frac{\partial}{2}\right)-8 F_{A}\left(\frac{q i_{v}}{2 M}\right) \sin \frac{\theta}{2}\right\} .
\end{array}
$$

In the approximation $q^{2}=v^{2}+k^{2}-2 v k \cos \theta \simeq 2 v^{2}$ $(1-\cos \theta)$, i.e. $\omega=0$, the angular distribution can be integrated to give

$\sigma_{V N}=\frac{G^{2}}{\pi} k E\left[1+3 F_{A}^{2}+\frac{20}{3}\left(\frac{V \mu_{v}}{2 M}\right)^{2}-16 F_{A}\left(\frac{V \mu_{v}}{2 M}\right)\right]$.

These last two terms are usually neglected at low neutrino energies, however, at $\nu=53 \mathrm{MeV}$ the last term contributes +2.6 . Th1s cross section, evaluated with the coupling constants,

$$
G=1 \times 10^{-5} / M_{\mathrm{P}}^{2}, \quad F_{A}=-1.24
$$

is show in Fig. (1) in the region of electronneutrino energles avallable from the decay of stopped muons:

$$
\mu^{+}+e^{+}+\bar{v}_{\mu}+v_{e}
$$

The cross section for the breakup of the deuteron

$$
v_{e}+D \rightarrow P+P+e^{-}, Q=-1.44 \mathrm{MeV}
$$

has a density of final states

$$
\frac{d n}{d \nu}=\frac{d^{3} k}{(2 \pi)^{3}} \frac{d^{3} p}{(2 \pi)^{3}} \frac{1}{d \nu}=\frac{k E d \Omega e}{(2 \pi)^{3}} \frac{M p}{2(2 \pi)^{3}} d E d \Omega p
$$

where $p$ is the relative momentum between the two final protons

$$
V=E+\frac{p^{2}}{M}+Q
$$

The nuclear recoil energy has been neglected. 


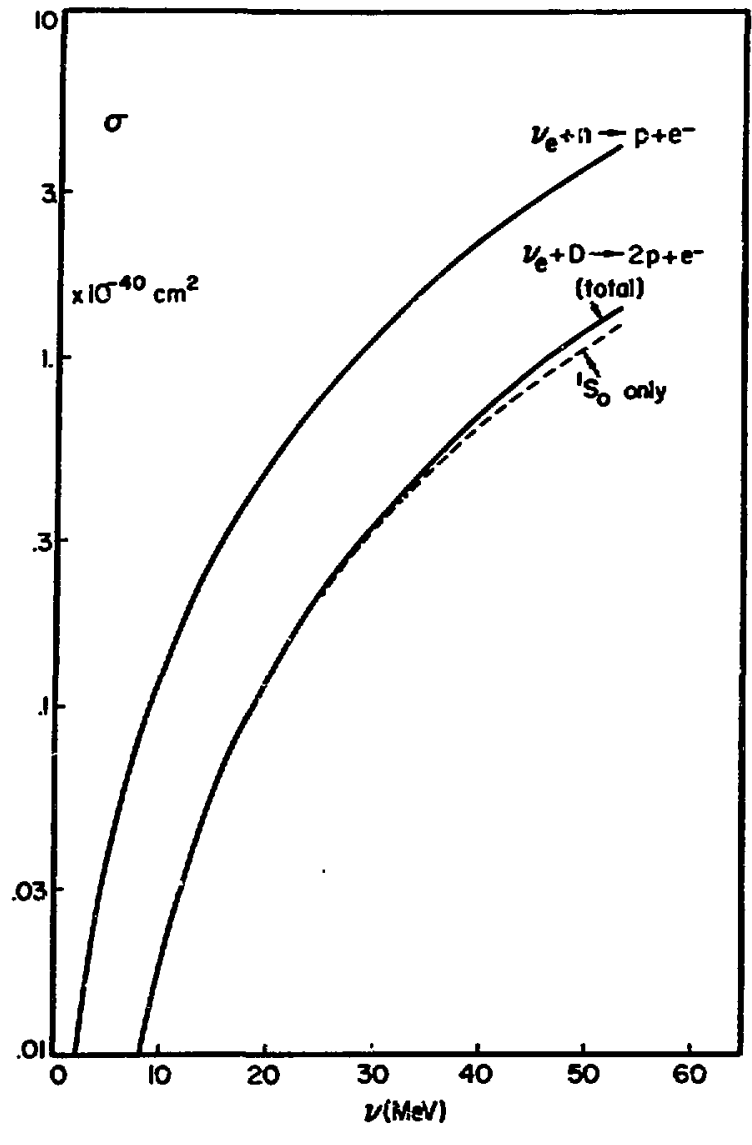

FIg. 1. Electron-neutrino reacticn cross section for the free neutron and the deuteron. The dashed line is for the singlet s-wave final state while the solid line includes the contribution of higher partial waves.

The main transition at low Iowentum transfer $1 \mathrm{~s}$ the ${ }^{3} S_{1} \rightarrow{ }^{1} S_{0}$ Induced by the operators $T_{1}^{e 15}$ and $T_{1}^{\text {wrag }}{ }^{1}$ (The operator $H_{0}$ or $L_{0}$ would induce ${ }^{3} S_{1} \rightarrow{ }^{3} S_{1}$ which is forbidden for identical particles in the final state.) By angular momentum recoupling one can show

$$
\begin{aligned}
& \left\langle{ }_{0} s_{0} \| T_{1}^{e 1_{5}} \text { or mag } \mid{ }^{3} s_{1}\right\rangle \\
& =\frac{1}{\sqrt{2}}\left\langle\frac{1}{2} \| T^{e 1_{5}} \text { or mag }|| \frac{I}{2}\right\rangle I_{0}(p, q) \\
& I_{0}(p, q)=\int_{0}^{\infty} d^{3} r \psi_{p}(r) J_{0}\left(\frac{q r}{2}\right) \psi_{0}(r)
\end{aligned}
$$

where $\psi_{0}$ and $\psi_{p}$ are the initial and final s-wave radial wave functions of the bound deuteron and the continuum protons. The double differential cross section obtained by integrating over the (isotropic) proton angular distribution is

$$
\begin{aligned}
\frac{d^{2} \sigma_{V D}}{d \Omega_{e} d E} & =\frac{4}{3} \frac{G^{2}}{(2 \pi)^{2}} k E \frac{M p}{(2 \pi)^{2}} \\
& \times\left\{\left(\frac{1}{2}+\frac{1}{2} \sin ^{2} \frac{\theta}{2}\right)\left[F_{A}^{2}+\left(\frac{q \mu_{v}}{2 M}\right)^{2}\right]\right. \\
& -2 \sin \frac{\theta}{2} F_{A}\left(\frac{q \mu_{v}}{2 M}\right) I_{O}{ }^{2}(p, q) .
\end{aligned}
$$

Effective-range theory provides simple, rellable wave functions for the ${ }^{3} \mathrm{~S}_{1}$ bound state and the is continuum state

$$
\begin{aligned}
\psi_{0}(r) & =\sqrt{\frac{2 \alpha}{I-\alpha r_{t}}} \frac{e^{-\alpha r}}{r} \frac{1}{\sqrt{4 \pi}} \\
\psi_{p}(r) & =\frac{\sin \left(p r+\delta_{s}\right)}{p r} \\
p \cot \delta_{s} & =-\frac{1}{a_{s}}+\frac{1}{2} \cdot s p^{2}
\end{aligned}
$$

where $\alpha=.232 \mathrm{fm}^{-1}, \mathrm{r}_{\mathrm{t}}$ (triplet effective range) $=$ $1.75 \mathrm{fm}, r_{s}=2.79 \mathrm{fm}$, and $a_{s}$ (singlet scattering length for protions) $=-7.82$. These functions give

$$
\begin{aligned}
I_{0}^{e r}(p, q) & =\sqrt{L \pi} \sqrt{\frac{2 \alpha}{1-\alpha r_{t}}} \frac{1}{p q / 2} \\
& x\left\{\frac{\cos \delta_{s}}{4} \ln \frac{\alpha^{2}+(p+q / 2)^{2}}{\alpha^{2}+(p-q / 2)^{2}}+\frac{\sin \delta}{2}\right. \\
& {\left.\left[\tan ^{-1} \frac{2 \alpha(q / 2)}{\alpha^{2}+p^{2}-(q / 2)^{2}+E \pi}\right]\right\} } \\
& =0 \text { for } \alpha^{2}+p^{2}-(q / 2)^{2} \geq 0 \\
& =1 \text { for } \alpha^{2}+p^{2}-(q / 2)^{2}<0 .
\end{aligned}
$$


The total cross section, obtained by numerically integrating over the electron energy and angular distribution, is shown in Fig. (1).

The electron-neutrino spectrum from mu-plus decay at rest is given (with $\cup$ in MeV) by

$$
\frac{d N}{d v}=\frac{12}{(53)^{4}} v^{2}(53-v)
$$

The product $\frac{d N}{d v} \times \sigma_{U D}$ is plotted in FIg. (2) showing $v=43 \mathrm{MeV}$ as the most probable interaction energy. When $\sigma_{U N}$ and $\sigma_{U D}$ are averaged over the neutrino spectrum we obtain

$\left\langle\sigma_{v N}\right\rangle=1.52 \times 10^{-40} \mathrm{~cm}^{2},\left\langle\sigma_{v D}\right\rangle=.44 \times 10^{-40} \mathrm{~cm}^{2}$.

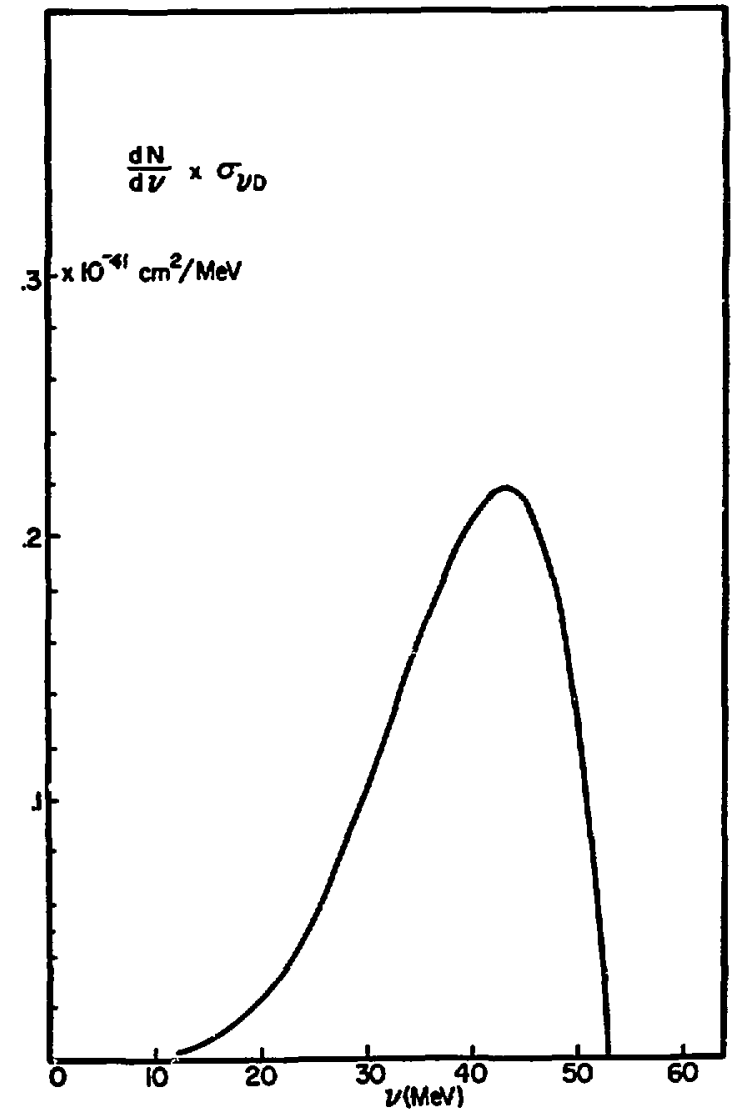

F1g. 2. The product of the deuteron (S-wave) cross section and the electron-neutrino spectrum from stupped mu-plus decays.
The electron energy spectrum at $\nu=43 \mathrm{MeV}$ is shown in Fig. (3). The most probable election energy ( $41.2 \mathrm{MeV}$ ) is close to the maximum available because of the phase space weighting and the preference of the two outgoing nucleons for low relative energies. The weighted angular distribution of electrons is shown in Fig. (4) inuicating a strong fore-aft asymmetry when the weak magnetism term is taken into ascount.

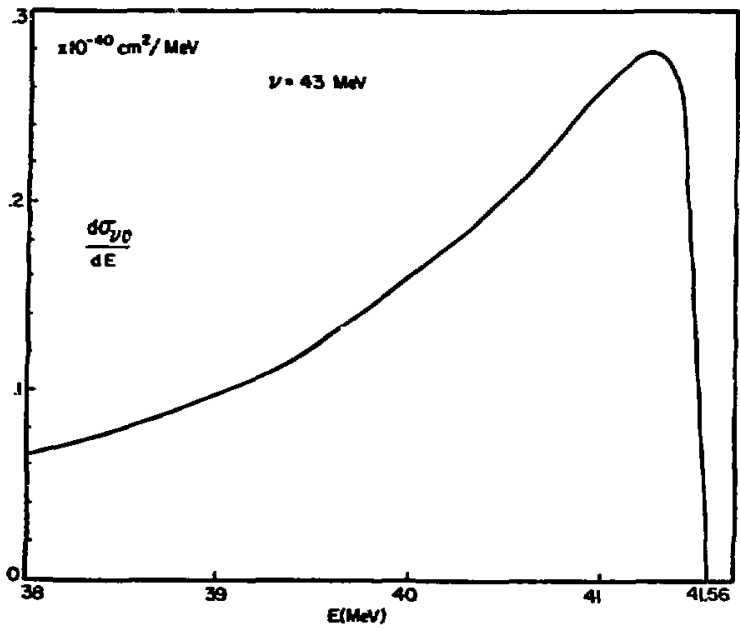

Fig. 3. Energy spectrum of electrons produced by 43-MeV neutrinos.

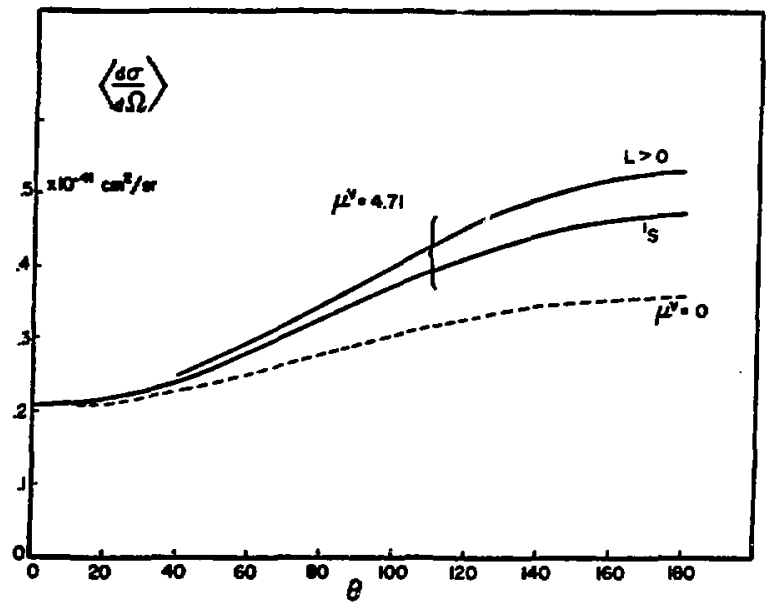

Fig. 4. Angular distribution of all electrons averaged over the neutrino spectrum. The curves show the effects of the magnetic moment term and higher partial waves. 
The contribution of higher nucleon partial waves can be obtained by adding the $\mathrm{L}=$ even singlet partial waves using

$$
\psi_{p}=\frac{1}{2}\left(e^{i \vec{p} \cdot \vec{r}}+e^{-i \vec{p} \cdot \vec{r}}\right)-j_{0}(p r)
$$

and the $\mathrm{L}=$ odd triplet partial waves using

$$
\psi_{p}=\frac{1}{2}\left(e^{1 \vec{p} \cdot \vec{r}}-e^{-i \vec{p} \cdot \vec{r}}\right)
$$

The cross section is augmented by these higher partial waves at the 'igher neutrino energies and back angles as shown in Figs. (1) and (4). The spectrum averaged cross section for the deuteron increases to

$$
\left\langle\sigma_{v D}\right\rangle_{\text {total }}=.48 \times 10^{-4 \theta} \mathrm{cm}^{2}
$$

The present calculation differis from the extension of the Kelly and Überall calculation ${ }^{3}$ by Chen ${ }^{4}$ in that the momentum dependence of the radial integral has been taken into account and the weak magnetism term and higher partial waves added.

The spectrum-averaged cross sections for the free neutron and deuterium can be compared to the neutrino-electron elastic scattering and to recent shell model calculations for carbon ${ }^{1}$ and oxygen ${ }^{5}$.

$$
\begin{aligned}
& \left\langle\sigma_{v e}\right\rangle=.0053 \times 10^{-40} \mathrm{~cm}^{2} \\
& \left\langle\sigma_{v C}\right\rangle=.146 \times 10^{-40} \mathrm{~cm}^{2} \\
& \left\langle\sigma_{v 0}\right\rangle=.052 \times 10^{-40} \mathrm{~cm}^{2}
\end{aligned}
$$

These results show that the deuteron is an Important target for neutrino interactions because of 1ts large reaction cross section and because this cross section can be rellably computed. The proposed LAMPF experiment of Hughes, Nemethy, Duclos, Burman, and Cochran will utilize a $6 \mathrm{~m}^{3}$ deuteratedwater Cerenkov counter separated by $6 \mathrm{~m}$ of shielding from the LAMPF beam stop. With $1 / 3 \mathrm{~mA}$ of $750 \mathrm{MeV}$ protons incident on the beam stop a $v_{e}+D \rightarrow 2 P+e^{-}$ event rate of 40 per day can be expected for the deuteron spectrum averaged cross section.
The deuteron cross section is closely related to the weak reaction

$$
P+P \rightarrow D+e^{+}+v_{e}
$$

which governs the rate of proton fusion in the sun and other stars. This reaction, a ${ }^{1} S_{0} \rightarrow{ }^{3} S_{1}$ transition, has never been observed in the laboratory. Therefore, a measurement of the neutrino disintegration of the deuteron will help our understanding of the astzophysical process.

\section{References}

1. J. S. O'Connell, T. W. Donnelly and J. D. Walecka, "Semi-leptonic Weak Interactions with C'," Phys. Rev. C, 6, 719 (1972).

2. J. D. Walecka, "Semi-leptonic Weak Interactions in Nucle1" for the book Muon Physics, edited by v. W. Hughes and C. S. Wu, Stanford preprint IIP-39i.

3. F. J. Kelly and H. Übera11, Phys. Rev. Letters 16. 145 (1966).

4. H. H. Chen, University of Californta Irvine, Internal Report UCI-10P19-58.

5. T. W. Donnelly, private communication. 